\title{
The Emerging Epidemic of Nonalcoholic Fatty Liver Disease and Cardiovascular Risk: True, True, and Related?
}

\author{
Jordan E. Morningstar ${ }^{1}$. Wing-Kin Syn ${ }^{1,3,4} \cdot$ Sheldon E. Litwin ${ }^{2,3}$
}

Published online: 5 February 2020

(c) Springer Science+Business Media, LLC, part of Springer Nature 2020

\section{Introduction}

Nonalcoholic fatty liver disease (NAFLD), a condition that affects over 30\% of Americans, is one of the leading indications for liver transplantation. The prevalence of NAFLD has paralleled the rising rates of obesity and the metabolic syndrome. One study found that the prevalence of NAFLD was over seven times higher in patients who were obese compared to those with normal weight [1]. Since several studies have demonstrated an association between NAFLD and an increased risk of cardiovascular events, it has been proposed that NAFLD may be an independent risk factor for atherosclerosis [2]. Although it is difficult to dissect the association between NAFLD and cardiovascular disease (CVD) due to the frequent presence of shared risk factors (i.e., obesity, diabetes, and dyslipidemia), NAFLD, and in particular the more advanced condition of nonalcoholic steatohepatitis (NASH) with fibrosis, is associated with an increased risk of CVD that is independent of traditional risk factors [2]. Other studies dispute this finding, suggesting that CVD in NAFLD patients is solely due to a higher prevalence of acknowledged traits that increase cardiovascular risk [3] Regardless of the underlying mechanisms, CVD is thought to be the leading cause of death in patients with NAFLD [4].

Sheldon E. Litwin

litwins@musc.edu

1 Division of Gastroenterology and Hepatology, Department of Internal Medicine, Medical University of South Carolina, Charleston, SC, USA

2 Division of Cardiology, Department of Internal Medicine, Medical University of South Carolina, 114 Doughty Street, Charleston, SC 29412, USA

3 Ralph H Johnson Veterans Affairs Medical Center, Charleston, SC, USA

4 Department of Physiology, Faculty of Medicine and Nursing, University of Basque Country UPV/EHU, Leioa, Spain
In the current issue of Digestive Diseases and Sciences, Labenz et al. identified an association between NAFLD and CVD in a large administrative database of primary care practices in Germany [5]. In this study, CVD was defined as incident myocardial infarction (MI), coronary heart disease (CHD), atrial fibrillation (AF), and stroke. In a sample of 22,048 patients with a diagnosis of NAFLD compared with a matched cohort without this diagnosis, they demonstrated that the association between NAFLD and CVD was present despite matching for covariates including age, sex, diabetes, hypertension, and hyperlipidemia. Although statistically significant, the absolute risk was fairly modest with $2.9 \%$ incidence of MI in the NAFLD group versus $2.3 \%$ in the control group. The authors pointed out that although NAFLD is estimated to have a prevalence of $25 \%$ in Germany, just $3 \%$ of the analyzed cohort had a diagnosis of NAFLD, highlighting the underrecognition of NAFLD in existing healthcare systems and/or problems with administrative databases. The article raises a number of important questions regarding NAFLD and CVD.

\section{Is Population-Level Screening for NAFLD Feasible?}

There is controversy regarding the utility of routine screening for NAFLD, related to uncertainty about the accuracy of different screening approaches, the cost-effectiveness of screening and a current lack of established treatments for the disorder. Although screening is not part of the current American Association for the Study of Liver Diseases (AASLD) guidelines, recommendations published by the European Liver and Diabetes Society in 2016 and the American Diabetes Association in 2019 suggest that routine screening be implemented [6, 7]. Several proposed algorithms exist to screen for NAFLD, although no current consensus exists [8]. For example, although serum levels of the aminotransferases ALT and AST have been used to screen for NAFLD, levels 
of these markers may be normal in up to $80 \%$ of patients with established NAFLD [9]. Furthermore, elevated AST or ALT levels do not provide information about the stage of NAFLD (i.e., NASH, fibrosis, cirrhosis), or its potential for progression [9]. As such, these markers are not recommended for routine screening for NAFLD. Imaging tests, including ultrasound, computed tomography (CT), and conventional magnetic resonance imaging (MRI), while able to detect steatosis, are not sensitive or specific for NASH and fibrosis, and cannot accurately distinguish the stage of liver fibrosis. Further, these technologies, particularly CT and MRI, are impractical to implement as screening modalities due to their expense and unknown cost-effectiveness. Although more specialized techniques such as MR elastography (MRE) offer improved sensitivity and specificity compared with traditional imaging, they are expensive, not widely available at this time, and are therefore impractical for use in screening. Transient elastography techniques, such as Fibroscan ${ }^{\mathrm{TM}}$ offer high sensitivity and specificity, are noninvasive, relatively quick, and inexpensive, and therefore could potentially be employed for routine screening. While the Fibroscan ${ }^{\mathrm{TM}}$ is already in use in parts of Europe, additional evaluation of its utility and cost-effectiveness is needed. The AASLD currently recommends assessing likelihood of advanced disease using two different risk scores: the NAFLD fibrosis score (NFS) is based on six readily available parameters including age, body mass index (BMI), hyperglycemia, platelet count, albumin, and AST/ALT ratio. The FIB4 score is a composite of four parameters (platelet count, age, AST, and ALT). The NFS can predict advanced fibrosis with an area under the receiving operating curve of 0.85 [8], and another study demonstrated that the NFS and FIB4 were comparable to MRE and transient elastography in the prediction of advanced fibrosis [10]. Nonetheless, these screening tools are probably best used to identify those who are unlikely to have advanced liver disease (i.e., the negative predictive values are significantly higher than the positive predictive values) and thereby reduce need for more expensive referral to specialty care and advanced evaluation techniques. Implementation of a readily available, reliable, and inexpensive noninvasive test with high positive predictive value remains a high priority.

\section{Does NAFLD Independently Increase the Risk of Cardiovascular Disease?}

It has proven difficult to tease out whether the high risk of CVD in patients with NAFLD is due to shared risk pathways, or due to an independent relationship between the metabolic dysregulation and inflammation seen in NAFLD. Labenz et al. argue that the risk of CVD in patients with NAFLD is independent of other risk factors. Similar findings have been demonstrated in some, but not all previous studies on the topic. In particular, a study with similar design comparing 86,098 patients with NAFLD or NASH to 4,664,988 matched controls from four European countries was not associated with an increased risk for MI or stroke [3]. The interplay of NAFLD and CVD is complicated by the fact that increasing stages of liver fibrosis appear to be associated with increasing carotid artery intimal medial thickness, a marker of atherosclerotic cardiovascular disease [2]. At present, there is still uncertainty as to whether, or how strongly, the presence and severity of NAFLD are linked to cardiovascular risk.

\section{What Is Optimal Management of Cardiovascular Risk in NAFLD?}

Screening for NAFLD in primary care patients may present an opportunity to identify patients who require more aggressive cardioprotective therapies. On the other hand, a case can be made for aggressive risk factor modification via lifestyle or pharmacological therapy in all patients with obesity, insulin resistance, diabetes, and hyperlipidemia regardless of the presence of NAFLD. Current cardiovascular risk assessment tools such as the Framingham risk score and the ASCVD risk score do not take NAFLD, NASH, or obesity into account and may not capture the increased cardiovascular risk present in this population. It will be important to study whether presence or severity of NAFLD or NASH can be incorporated into these or other risk assessment strategies.

Lifestyle measures, especially weight management, are the hallmark of treatment for patients with NAFLD. Weight loss of five percent has been associated with a reduction in steatosis of approximately $30 \%$, whereas weight loss of 9.3\% was associated with an improvement in the NAFLD activity score (NAS), a measurement of histologic markers of inflammation and necrosis; a different study found that weight loss of $>10 \%$ was associated with lower levels of fibrosis [4]. Bariatric surgery improves histologic parameters in NAFLD, although reductions in inflammation and fibrosis were not seen [11]. Thus, all overweight or obese patients with NAFLD should be offered intensive lifestyle interventions with a goal of $10 \%$ body weight loss.

There are only limited data on the use of pharmacological therapies to treat NAFLD and/or the associated cardiovascular risk. Patients with type 2 diabetes given pioglitazone, a thiazolidinedione anti-diabetes medicine had decreased liver fibrosis, improved adipose tissue insulin sensitivity, and reduced number of cardiovascular events [4]. Nevertheless, this therapy has also been associated with weight gain and questionable improvements in CVD outcomes. A recent double-blind, placebo-controlled phase 2 study demonstrated that patients who received the glucagon-like 
peptide 1 receptor agonist liraglutide had a statistically significant reduction of biopsy-proven NASH compared with placebo [12]. A different study demonstrated that 24 weeks of treatment with the sodium-glucose co-transporter 2 inhibitor dapagliflozin reduced several markers of liver fibrosis [13]. Since weight loss improves histologic parameters of NAFLD, weight loss-inducing anti-diabetic medications may theoretically be helpful for preventing or reducing severity of NAFLD. Nonetheless, there are no current trials testing this hypothesis using FDA-approved weight loss pharmacotherapies. Finally, emerging antifibrotic medicines are being developed to treat individuals with NASH fibrosis and may soon be approved by the FDA. Future studies will be needed to determine if treatment of NAFLD fibrosis leads to subsequent improvements in cardiac outcomes.

\section{Conclusions}

The study by Labenz et al. reinforces the fact that NAFLD remains very significantly underdiagnosed or under-reported in the general population. The data from this study support the hypothesis that NAFLD is independently associated with CVD. These new data do not give guidance on the utility or cost-effectiveness of universal screening for NAFLD. Newer risk scoring systems and less expensive, noninvasive, and more reliable methods of NAFLD diagnosis will make screening more feasible to implement on a population scale. Yet, the benefits of identifying NAFLD will not become evident until effective treatments are identified. The burden of proof lies with the growing ranks of the new cardiometabolic field.

\section{References}

1. Yki-Järvinen H. Non-alcoholic fatty liver disease as a cause and a consequence of metabolic syndrome. Lancet Diabetes Endocrinol. 2014;2:901-910.

2. Targher G, Day CP, Bonora E. Risk of cardiovascular disease in patients with nonalcoholic fatty liver disease. $N$ Engl J Med. 2010:363:1341-1350.
3. Alexander M, Loomis AK, van der Lei J, et al. Non-alcoholic fatty liver disease and risk of incident acute myocardial infarction and stroke: findings from matched cohort study of 18 million European adults. BMJ. 2019;367:15367-15367. (Clinical research ed.).

4. Stefan N, Häring H-U, Cusi K. Non-alcoholic fatty liver disease: causes, diagnosis, cardiometabolic consequences, and treatment strategies. Lancet Diabetes Endocrinol. 2019;7:313-324.

5. Labenz X, Huber C, Michel Y, et al. Impact of NAFLD on the incidence of cardiovascular diseases in a primary care population in Germany. Dig Dis Sci. (Epub ahead of print). https://doi. org/10.1007/s10620-019-05986-9.

6. European Association for the Study of the Liver (EASL), European Association for the Study of Diabetes (EASD), European Association for the Study of Obesity (EASO). EASL-EASDEASO clinical practice guidelines for the management of nonalcoholic fatty liver disease. J Hepatol. 2016;64:1388-1402.

7. American Diabetes Association. Comprehensive medical evaluation and assessment of comorbidities: standards of medical care in diabetes. Diabetes Care. 2019;42:S34.

8. Chalasani N, Younossi Z, Lavine JE, et al. The diagnosis and management of nonalcoholic fatty liver disease: practice guidance from the American Association for the Study of Liver Diseases. Hepatology. 2018;67:328-357.

9. Browning JD, Szczepaniak LS, Dobbins R, et al. Prevalence of hepatic steatosis in an urban population in the United States: impact of ethnicity. Hepatology. 2004;40:1387-1395.

10. Imajo K, Kessoku T, Honda Y, et al. Magnetic resonance imaging more accurately classifies steatosis and fibrosis in patients with nonalcoholic fatty liver disease than transient elastography. Gastroenterology. 2016;150:626-637.e7.

11. Klein S, Mittendorfer B, Eagon JC, et al. Gastric bypass surgery improves metabolic and hepatic abnormalities associated with nonalcoholic fatty liver disease. Gastroenterology. 2006;130:1564-1572.

12. Armstrong MJ, Gaunt P, Aithal GP, et al. Liraglutide safety and efficacy in patients with non-alcoholic steatohepatitis (LEAN): a multicentre, double-blind, randomised, placebo-controlled phase 2 study. The Lancet. 2016;387:679-690.

13. Shimizu M, Suzuki K, Kato K, et al. Evaluation of the effects of dapagliflozin, a sodium-glucose co-transporter-2 inhibitor, on hepatic steatosis and fibrosis using transient elastography in patients with type 2 diabetes and non-alcoholic fatty liver disease. Diabetes Obes Metab. 2019;21:285-292.

Publisher's Note Springer Nature remains neutral with regard to jurisdictional claims in published maps and institutional affiliations. 